\title{
The Promotion of Multilingualism in Albania: The Role of Italian Language at School and in the Work-Place
}

\author{
Dr.Svjetllana Titini
}

\author{
Università "Aleksandër Moisiu" Durazzo, Albania
}

Doi:10.5901/ajis.2013.v2n9p130

\section{Abstract}

Languages have a very significant role in society, in economy, in the working place and in the political relationships among different countries. Multilingualism is a key element nowadays that not only facilitates communication but helps to move between cultures. This article aims to present the situation of multilingualism in Albania with reference to the promotion of language skills in school and their role on employability in the labor market by focusing on the ltalian language as one of the best known and most popular languages among European ones that are spoken in Albania.Reforms in the curricula of the education system in Albania are conceptualized according to the education strategies of the Council of Europe and of the EU where Albania aims to become a member state- and focuses on the promotion of multilingualism and multiculturalist education among the new generation. It is in such context that the teaching and learning of community languages became part of the curricula starting from the very first classes in the elementary school and adding to two the number of languages that are taught in this phase of education in addition to the mother tongue. In the course of secondary education there is assigned an important role to foreign language which are part of the curricula and of the maturity exam; meanwhile their importance is clearly confirmed during university studies and graduate studies. The practice in job recruitment provides evidence that the knowledge of languages increases considerably the possibilities and chances of success for those aspiring to find work in both public and private sectors. Knowledge of a foreign language (preferably English) is now one of the key requirements for participation in all competitions and job applications. In addition, the knowledge of multiple languages is a credit to those who participate in these contests. Even in the private sector the knowledge of languages has become a necessity both in the context of communication and trade with foreign companies and suppliers, and in the area of the work carried out on behalf of foreign companies that conduct their business in Albania. In conclusion, there will be shown that the Italian language occupies an important place in the system of education and training, as well as having a central role in the labor market, especially in the private sector, while taking into account the considerable number of Italian companies present on the territory of Albania and the intensity of trade and cultural exchanges between these two countries, Albania and Italy.

\section{Introduzione}

Le lingue ricoprono un ruolo importante nella società, nell'economia, nel mondo del lavoro e nelle relazioni politiche fra gli stati costituendo parte integrante della cultura e della civiltà umana. La conoscenza di un certo numero di lingue straniere, al giorno d'oggi, è un elemento chiave che non si limita a facilitare la comunicazione ma contribuisce a muoversi fra le culture.

Il valore della diversità linguistica come patrimonio da tutelare e rispettare e la funzione di integrazione sociale, economica e politica che viene riconosciuto alle lingue sono enunciati in essenziali documenti europei i quali oltre a ribadire il multilinguismo come uno dei tratti caratterizzanti dell'identità europea hanno dato voce all'esigenza di elaborare politiche educative plurilingue in tutta l'Europa.

A differenza del multilinguismo che può essere considerato come proprietà di un determinato territorio, il plurilinguismo viene considerato proprietà del singolo individuo che si esprime nella capacità umana di usare le lingue per comunicare e partecipare all' interazione culturale, disponendo di vari tipi di competenza in più lingue (o varietà di lingua) ed avendo esperienza di più culture (Council of Europe, 2001).

A partire da queste considerazioni, questo articolo si propone di presentare la situazione del plurilinguismo in Albania con riferimento alla promozione delle competenze linguistiche a scuola e il loro ruolo sull'occupabilità nel mercato del lavoro ponendo l'attenzione sulla lingua italiana in quanto una delle lingue comunitarie più conosciute e parlate nel paese. 


\section{II plurilinguismo e l'educazione plurilingue}

La valorizzazione e la promozione del plurilinguismo è diventata progressivamente l'asse di un modello di politica linguistica originale dell'Europa che ha come finalità di «incoraggiare le persone al rispetto e all'apertura verso la diversità delle lingue e delle culture in una società multilingue e multiculturale e di promuovere la consapevolezza della dimensione delle loro capacità e del loro potenziale di sviluppo. Un efficace apprendimento di una 0 più lingue, la consapevolezza del valore della diversità e dell'alterità e il riconoscimento dell'utilità di ogni competenza, anche parziale, sono necessari ad ogni individuo per esercitare, come membro attivo di una comunità sociale, la sua cittadinanza democratica in una società multilingue e multiculturale».(Council of Europe, 2010, p.27)

L'approccio plurilingue del quale si è parlato esplicitamente nel QCER è compito precipuo della scuola che contribuisce alla formazione delle competenze linguistiche attraverso l'insegnamento plurilingue ed è ancora compito delle politiche educative di sviluppare una coscienza pluriculturale fondata sulla disponibilità di imparare e di utilizzare anche parzialmente le lingue degli altri e sulla sensibilità verso altre comunità linguistiche culturali in modo tale che gli individui si trovino in condizione di agire sulla base del rispetto reciproco e dell'inclusione.

Come segnalato nella Guida per lo sviluppo e l'attuazione di curricoli per una educazione plurilingue e interculturale tale approccio di apertura alla diversità linguistica e culturale «ha un orientamento inclusivo ed interculturale perché mette al centro della riflessione le (varietà di) lingue parlate dai bambini(alunni) trasformandole da "strumenti" quotidiani d'uso in quelli che sono per loro "oggetti (degni) di riflessione" e conferendo loro così una visibilità ed una legittimità scolastiche che sono alla base di una vera loro valorizzazione». (Council of Europe, 2010, p.152)

In conseguenza al centro dell' azione educativa deve essere l'apprendente e lo sviluppo del suo repertorio plurilingue e pluriculturale attraverso l'offerta diversificata in lingue da parte della scuola.

In un'epoca contraddistinta da nuove dimensioni transnazionali dell'economia, del sistema organizzativo e della comunicazione l'educazione plurilingue valorizza il rispetto e l'apertura alla diversità delle lingue e delle culture attorno a noi ai fini della costruzione di una società inclusiva e plurale. II plurilinguismo individuale inteso come riconoscimento della pluriappartenenza e pluralità delle identità si trasforma in un punto di forza e vantaggio per il singolo individuo e per la comunità nel suo complesso.

Sulle linee guida tracciate da questa piattaforma della politica europea sull' educazione plurilingue che emerge dai vari testi si è costruito, dal Ministero dell'Istruzione e della Scienza in Albania, la politica della promozione delle lingue straniere nel sistema dell'istruzione nazionale esposta nella Strategia Nazionale sulla Formazione Parauniversitaria, nella legge sul sistema dell' istruzione parauniversitaria nella Repubblica d'Albania, nella legge sull'istruzione superiore nella Repubblica d'Albania e nella riforma dei curricula realizzata negli ultimi anni.

Nel primo di questi importanti documenti sui quali si fonda l'organizzazione e il funzionamento del sistema dell'istruzione albanese si sottolinea l'obbiettivo che al termine della scuola secondaria superiore gli alunni siano in grado di comunicare in almeno due lingue straniere e di raggiungere il livello $B_{1}$ nella conoscenza della prima lingua. La legge sul sistema dell' istruzione parauniversitaria specificando l'inserimento dello studio della lingua straniera nei curriculum della scuola albanese già dalla terza elementare prevede la lingua straniera come materia di esame sia negli esami nazionali dell'istruzione base ${ }^{1}$, sia negli esami di maturità (denominata la Maturità Statale). Nella predetta legge l'importanza che viene data allo studio delle lingue straniere si esprime anche nell'elencare fra le prime delle competenze fondamentali acquisite dagli studenti al compimento degli studi nell'istruzione secondaria di secondo grado la comunicazione nelle lingue straniere oltre a quella nella lingua madre. La stessa legge ammette l'istruzione bilingue nel sistema dell'istruzione nazionale in base agli accordi bilaterali fra la Repubblica dell'Albania e gli altri paesi. Ulteriormente la Legge sull'istruzione superiore rende obligatorio l'esame che attesta la conoscenza dell'inglese sulla base dei test internazionali al termine del secondo e terzo ciclo degli studi (master e dottorato) e delle scuole di specializzazione.

\section{L'italiano nell' sistema scolastico albanese}

I rapporti di vicinato e soprattutto l'intensificazione degli scambi economici e culturali tra l'Albania e l'Italia, dopo gli anni '90, hanno contribuito a crescere l'interesse per la lingua italiana e a diffondere il suo insegnamento nelle scuole?

\footnotetext{
${ }^{1}$ L'istruzione base comprende l'istruzione primaria e l'istruzione secondaria di primo grado.

'Le fonti di riferimento per i dati riportati in seguito sono il Ministero dell'Educazione e della Scienza della Repubblica d'Albania e l'Ambasciata italiana a Tirana.
} 
Al compimento della politica sulla promozione delle lingue straniere, che segue il Ministero albanese dell' Istruzione e della Scienza(MASH), la lingua italiana è inserita nei curricoli ministeriali e gli studenti albanesi possono scegliere di studiarla come seconda lingua straniera dalla I classe della scuola media di ${ }^{\circ}$ grado 0 dalla $X$ classe del triennio della scuola media di $I^{\circ 3}$. Corsi curricolari d'italiano come seconda lingua straniera sono istituiti anche in diverse scuole private in vari distretti albanesi ${ }^{4}$.

Inoltre, sono cinque gli Atenei albanesi (Tirana, Scutari, Elbasan, Valona, Argirocastro) che offrono corsi di Laurea in italiano. Nell'a.a. 2012/2013, gli studenti universitari albanesi che studiano l'italiano a vario titolo sono 3.399 in totale, di cui 1.202 iscritti ai corsi di Laurea in Italiano, 1.186 che lo studiano come seconda lingua straniera (LS2) e 1.011 che studiano l'italiano in corsi di laurea in altre discipline (in particolare i corsi di laurea in infermieria e quelli in economia). L'insegnamento dell'italiano nelle università statali albanesi è stato promosso anche attraverso l'istituzione, presso alcune Facoltà, di nuovi corsi di laurea che hanno registrato notevole successo offrendo agli iscritti una duplice qualifica ${ }^{5}$.

Nelle università private invece, l'italiano è insegnato come seconda lingua straniera nel corso di laurea di qualche Facoltà (Universiteti Planetar i Tiranës). Un caso particolare è rappresentato dall'università "Nostra Signora del Buon Consiglio", l'unica che svolge quasi l'intera attività didattica in italiano. Questa università, istituita nel 2004, con sede a Tirana ed Elbasan, ha attivato sette corsi di laurea in convenzione con tre atenei statali italiani (Bari, Roma Tor Vergata e Milano), potendo così rilasciare diplomi universitari validi anche in Italia. II numero considerevole di studenti italiani oltre agli studenti albanesi che studiano in questa università e i 500 docenti italiani che vi tengono interi corsi, contribuiscono a renderla il più grande progetto culturale-universitario italiano all'estero.

Un ruolo importante nella diffusione dell'insegnamento dell'italiano è svolto dagli accordi bilaterali in questione tra Italia e Albania.

A partire dal 1998, in seguito al Memorandum d'Intesa firmato dai rispettivi governi è iniziato, in Albania, il percorso formativo delle Sezioni Bilingui che non solo offrono ai giovani una formazione culturale e scientifica che gli rende capaci di studiare sia nelle università albanesi che in quelle italiane ma anche abilitano al lavoro in un contesto europeo. Così i giovani possono contribuire a rafforzare ulteriormente i legami tra i due paesi e i due popolí.

II percorso formativo delle Sezioni Bilingui, volto ad assicurare solide basi umanistico-scientifiche, si sviluppa come segue:

- durata degli studi: 7 anni, di cui 4 anni nella scuola di base e 3 nel liceo;

- oltre il $50 \%$ delle ore di lezione sono svolte in lingua italiana; lo studio dell'italiano inizia dalla VI classe, con insegnanti italiani e albanesi;

- nel VIII e IX classe inizia lo studio della terminologia che aiuterà gli studenti nell'apprendimento delle discipline umanistiche e scientifiche che saranno successivamente insegnate in italiano.

- dalla X classe sono insegnate in italiano le seguenti discipline: Lingua e Letteratura italiana, Matematica e Fisica, Storia, Arte, Chimica e Biologia;

- in ogni sede di sezioni sono presenti tre docenti della scuola italiana, inviati dal Ministero degli Affari Esteri: 1 di Lingua Italiana, 1 di Lingua e Letteratura Italiana e 1 di Matematica e Fisica.

In uscita dalla XII classe di liceo lo studente sarà in grado di capire senza difficoltà la lingua italiana e di esprimersi in modo spontaneo, scorrevole e preciso. Ciò vuol dire che, secondo il quadro comune di riferimento europeo delle lingue, il grado di conoscenza della lingua italiana è pari al livello $\mathrm{C} 1$.

Gli studenti delle sezioni bilingui sono facilitati, con riguardo al proseguimento degli studi in Italia, in quanto possono iscriversi alle università italiane senza sostenere l'esame di Certificazione di Conoscenza della Lingua Italiana (CELI3) e i migliori alunni, iscritti presso le università italiane, ricevono borse di studio del Ministero Affari Esteri italiano.

\footnotetext{
3 L'inserimento dello studio della lingua italiana è stato esperimentato per la prima volta nel liceo "Çajupi" di Tirana nel 1991 per essere poi estesa nelle scuole di tutto il paese.

4 In tutto il territorio del Paese sono 18 le scuole private che al momento insegnano la lingua italiana come seconda lingua straniera.

${ }^{5}$ Gli iscritti al Corso di laurea in Storia e lingua italiana (Università di Argirocastro) e al Corso di laurea in Geografia e italiano (Università di Elbasan), in seguito al compimento degli studi, conseguono rispettivamente la duplice qualifica di "Insegnante di Storia e di Lingua Italiana" e "Insegnante di Geografia e di Lingua Italiana".

${ }^{6}$ Le Sezioni Bilingui sono operative nelle scuole medie superiori: "Asim Vokshi", "Ismail Qemali" a Tirana; "Faik Konica", "Themistokli Gërmenji" a Korça; "Shejnaze Juka", " 28 Nentori" a Scutari e nelle scuole medie inferiori: "P. Budi" e "Shkolla e Kuqe" di Tirana; "S. Qiriazi" e "Shkolla e Re" di Korça; "I. Qemali" e "Ndre Mjeda di Scutari.
} 
II programma "Illiria", avviato nel 2002, costituisce il secondo progetto più importante per l'insegnamento/apprendimento dell'italiano nelle scuole albanesi. Questo Programma che ha inizialmente consentito, nel sistema educativo pubblico, l'insegnamento/apprendimento dell'italiano come prima lingua straniera dalla III elementare all'ultimo anno della scuola secondaria di secondo grado, si è esteso in tutti i distretti del Paese (rispetto ai 19 precedenti).

In più il programma "Illiria" ha introdotto l'inclusione di moduli formativi di materie non linguistiche nei curricoli delle scuole di formazione professionali, anche in considerazione delle opportunità di occupazione degli studenti di queste scuole presso le imprese italiane in Albania.

In linea con l'obiettivo di diffondere l'insegnamento dell' italiano svolge la sua attività anche l'Istituto Italiano di Cultura che organizza corsi di lingua e cultura italiana secondo il Quadro Comune Europeo dal livello A1 fino al C2. Nel 2012, 1240 studenti hanno frequentato i corsi dell'IIC di Tirana, che si svolgono, non solo a Tirana, ma anche su tutto il territorio albanese in altre 15 città. Oltre ad essere sede convenzionata per gli esami di Certificazione dell'Italiano Lingua Straniera dell'Università di Perugia, dell'Università di Siena e dell'Università Roma 3 da quest'anno l'Istituto è anche sede di corsi e esami Ditals dell'Università di Siena e ha preparato i propri insegnanti all'ottenimento di questo diploma di didattica. L'Istituto organizza ugualmente corsi per bambini (7-10 anni), corsi per adolescenti (11-14 anni) e campi-scuola nel periodo estivo per queste fasce di èta.

Rilevanti sono i contributi, dalla parte italiana, per la formazione degli insegnanti albanesi d'italiano e la fornitura di materiale didattico per biblioteche e laboratori delle scuole albanesi. Si sottolinea anche la collaborazione per la diffusione dell'insegnamento dell'italiano con l'Associazione dei docenti albanesi d'italiano (APADI).

Non va dimenticato anche il sostegno del Governo italiano per gli atenei albanesi (Tirana, Scutari, Elbasan, Valona, Argirocastro) presso cui lavorano professori di ruolo italiani con funzioni di Lettore.

\section{L'italiano in Albania e il mondo di lavoro}

Lo studio dell italiano all'estero è dovuto a tre motivi fondamentali: "il suo destino di grande lingua di cultura, il suo destino di lingua che si appoggia a una economia, nonostante tutto, in grande espansione, il suo destino come lingua delle comunità italiane all'estero" (Baldelli 1987, 25). In Albania, fuori dal essere una lingua conosciuta per semplici motivi culturali e storici, l'italiano sia largamente appreso per motivi di lavoro.

Come emerge dai dati l'Italia si colloca in cima della classifica per il numero delle imprese con capitale partecipato(risultano oltre 500 aziende italiane e joint venture italiano-albanesi concentrate in grande parte nella zona di Tirana e per il resto lungo la costa adriatica che operano nel settore edile, nel settore tessile e calzaturiero, nel commercio e nell'industria agro-alimentare.

Negli ultimi anni sono presenti sul mercato gruppi idustriali di grandi e medie dimensioni nei settori dell'energia e delle infrastrutture. Nel settore bancario svolgono la loro attività due grandi gruppi bancari italiani, (Intesa SanPaolo e il Gruppo Veneto Banca) che sono un appoggio finanziario importante per l'attività delle imprese italiane. Interessante sviluppo sta avendo inoltre la delocalizzazione dei servizi attraverso, ad esempio, le presenze di alcuni gruppi italiani che hanno qui realizzato call-centers. Gruppi imprenditoriali italiani sono presenti anche nel settore sanitario e di consulenza legale.

Gli investimenti italiani in crescità in questo decennio hanno creato, in Albania, migliaia di posti di lavoro e hanno fatto sì che l'italiano abbia un ruolo considerevole come lingua di lavoro.

La conoscenza dell'taliano ha offerto buone opportunità di occupazione per molti giovani diplomati e laureati.

L'italiano è diventato lingua di lavoro, per gli albanesi, oltre che per la forte presenza delle imprese italiane in vari settori dell'economia del paese anche per l'attivita di formazione linguistica e professionale inerente l'implementazione dell'Accordo di cooperazione bilaterale fra i due paesi in materia di migrazione per lavoro.

Questo ha favorito l'ingresso in Italia di molti cittadini albanesi offrendo un occupazione alternativa a quella nel loro paese ma allo stesso tempo ha dato luogo ad un approccio ravvicinato con la lingua, la cultura e in generale con il sistema italiano.

\section{Conclusioni}

La promozione del plurilinguismo, come condizione della piena integrazione del cittadino nella società europea, costituisce un principio base del nuovo sistema educativo in Albania. La costruzione di un educazione plurilingue e interculturale, in questi venti anni di ristrutturazione del sistema scolastico, si pone il compito di costruire il modello del cittadino europeo che si riconosca come membro di una società multiculturale e plurilingue in cui lo sviluppo della 
competenza plurilingue oltre a creare la consapevolezza della diversità delle lingue e del loro valore permette di entrare in contatto con altre culture per conoscersi e accettarsi reciprocamente.

II plurilinguismo apre, quindi, all' individuo, nuove possibilità di formazione e crescita personale, di inclusione sociale e di occupazione e contribuisce all' esercizio della cittadinanza attiva anche oltre i confini del territorio nazionale.

In questo contesto di promozione del plurilinguismo, quale riconoscimento del carattere plurale della nostra società, l'italiano risulta la lingua piu studiata come seconda lingua scelta dagli alunni albanesi. Inoltre l'italiano ha rafforzato il suo ruolo nel sistema educativo albanese tanto come prima lingua straniera attraverso l'attivazione del Programma Illiria quanto con il percorso formativo bilingue e biculturale offerto dalle Sezioni Bilingui.

La diffusa conoscienza dell'italiano ha facilitato l'occupazione di una parte della popolazione attiva ma vienne ancora considerato un indubbio vantaggio insieme ad altri fattori che rendono il paese un mercato interessante per i futuri investimenti italiani.

\section{References}

Baldelli, Ignazio (1987), La lingua italiana nel mondo. Indagine sulle motivazioni allo studio dell'italiano, Roma, Istituto della Enciclopedia Italiana.

Guida per lo sviluppo e l'attuazione di curricoli per una educazione plurilingue e interculturale www.coe.int//dg4//inguistic/Source ISource2010_ForumGeneva/GuideEPI2010_IT.pdf

Le Cadre européen commun de référence pour les langues (CECR) et lélaboration de politiques linguistiques: défis et responsabilités . Vedi anche l'edizione italiana del CEFR (QCER): Quadro Comune Europeo di Riferimento per le lingue: apprendimento, insegnamento, valutazione, La Nuova Italia-Oxford, Firenze, 2002. 